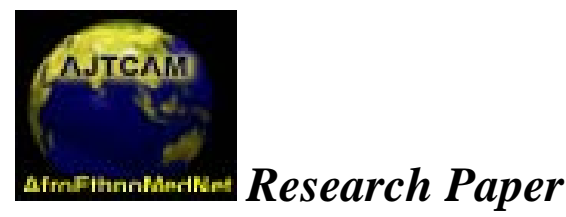

Afr. J. Infect. Diseases www.africanethnomedicines.net

\title{
ANTIMICROBIAL ACTIVITY OF COLEUS AROMATICUS (BENTH) AGAINST MICROBES OF REPRODUCTIVE TRACT INFECTIONS AMONG WOMEN
}

\author{
R. Annie Pritima ${ }^{1 *}$ and R. Selvaraj Pandian ${ }^{2}$ \\ Post-graduate and Research Department of Zoology, \\ The American College, Madurai - 625002, India. \\ * E-mail: godblesspreity@yahoo.com
}

\begin{abstract}
Coleus aromaticus (Benth) is a commonly available medicinal herb in India. The antimicrobial activity of this herb is tested as a cure for reproductive tract infections (RTI) among women. Fresh leaf has been used as a disc in different diameters to test the antimicrobial activity, against RTI causing microbes. Candida krusei showed the highest zone of inhibition of growth, followed by Candida albicans, Proteus mirablis, Escherichia coli, Staphylococcus aureus , Enterococcus faecalis, Klebsiella pneumoniae and the least inhibition was observed for Neisseria gonohorreae. Coleus aromaticus exhibits an effective antifungal and marked antibacterial activity. Using the leaf as a disc seems to be a novel and easy method that can be used initially to access the antimicrobial activity of a herb, before proceeding for further studies. This herb seems to be an ideal choice to treat RTI infections among women.
\end{abstract}

Key words: Coleus aromaticus, reproductive tract infections (RTI), leaf disc, herbs, microbes and antimicrobial activity.

\section{Introduction}

Bacterial vaginosis (BV), most commonly affects women of reproductive age .An alteration in the vaginal ecology in which Lactobacillus sp, the predominant organism in a healthy vagina is replaced by a mixed flora. The pathogenesis of BV may be similar to that of urinary tract infections, with the rectum serving as a reservoir for some BV associated flora. The organisms associated with BV have also been recognized as agents of female upper genital tract infection, including pelvic inflammatory disease, and some adverse outcomes of pregnancy, including premature rupture of membranes, fetal loss, postpartum endometritis and urinary tract infections (Spiegel, 1991).

Vaginitis is one of the most common complaints of women today. About $90 \%$ of patients with this problem suffer from infection of the vagina caused by Candida, Gardnerella or Trichomonas. The diagnosis and effective treatment of these common infections depend on accurate identification, effective specific therapy, and restoration of the normal ecosystem of the vagina. Proper hygiene habits, dietary control, and management of stress are all helpful factors in controlling recurrent vaginal infections (Friedrich , 1995 ).

A community based cross-sectional study of RTI, was conducted in 1996-1997 among married women 16-22 years of age in Tamil Nadu , India. Fifty-three percent of women reported gynecologic symptoms, 38\% had laboratory findings of RTI and 14\% had clinically diagnosed pelvic inflammatory disease or cervicitis. According to laboratory diagnoses, $15 \%$ had sexually transmitted infections and $28 \%$ had endogenous infections (Jasmin et al , 2005 ).

Many drugs including aspirin and ergot are of herbal origin. About one-quarter of the prescription drugs dispensed by community pharmacies in the US contain at least one active ingredient derived from 
plant material (Farnsworth and Morris,1976). The World Health Organization (WHO) estimates that 4 billion people, 80 percent of the world population use herbal medicine for some aspect of primary health care (Farnsworth et al ,1985) .

In Southern - Western Ghats of India (Madurai district, Tamil Nadu) an ethnobotanical survey was carried out to collect information on the use of medicinal plants. Information was gathered from paliyar tribes.The informants interviewed were 12 among whom 4 were tribal practitioners. A total of 60 ethno medicinal plant species distributed in 32 families were documented. The medicinal plants were used fresh, for the preparation of medicine. The documented ethnomedicinal plants were mostly used to cure skin diseases, poison bites, stomachache and nervous disorders (Ignacimuthu et al , 2006 ). Eighteen ethno medicinal plants and ethno botanical data were gathered from traditional healers residing in Palani hills of Southern Western Ghats, Tamilnadu , India .These plants were used in folk medicine, in the treatment of skin diseases, venereal diseases, respiratory problems and nervous disorders (Veeramuthu et al ,2006 ).

Antibiotics have been the only source of cure for RTI for a long time, the efficacy of antibiotics keeps on changing because of resistance. In preparing an antibiotic both pharmacodynamics and pharmacokinetics are considered very vital. The use of herbs or plant material as a potent antibiotic has always been of much debate .Herbs or plant materials (stem, bark, resin, leaf, flower, pollen, fruit, seed and root) find an important place in all forms of native medicinal therapy all over the world be it Ayurveda, Siddha , Unani , Homeopathy, Naturopathy, Oriental, or Native American Indian medicine. What is available readily in our hand is always of less importance and we want to go for what is not reachable and costly. Like the old saying goes history repeats itself, what was considered not so good, is making a come back and that too fully powered and is making the saying ," Your first aid box is in your kitchen" a fully true statement. Since RTI happens to be a delicate women's problem for which they seldom seek a doctor for treatment, because of social and psychological restrictions they have to face; especially in a developing country like India. Herbal cure, that is easily reachable for women would be a most welcome alternative. Locally available plant materials commonly used are found to have potential antibiotic properties.

Garlic (Allium sativum) showed higher diameter of inhibition zones for serotypes of Escherichia coli when compared to ciprofloxacin, chloramphenicol , tetracycline, streptomycin and nalidixic acid. Ginger (Zingiber officinale) extract showed moderate activity for the same (Indu et al ,2006). Onion (Allium серa) showed moderate antibacterial activity against Escherichia coli (Srinivasan et al , 2001 ). In this context, Coleus aromaticus, a commonly available household herb, has been studied to asses its antimicrobial activity.

\section{Methodology}

The plant chosen for study Coleus aromaticus (Benth), belonging to the Family : Lamiaceae is grown as a household herb in Tamilnadu (vernacular Tamil name: ommam or ommavalli) . This herb is native to East Indies and is widely cultivated in Africa and almost all tropical countries. It is also popularly known as "Indian Oregano ". It grows to a maximum of 1.5 to 2 meters and has a thick green stem. The leaves of the plant are thick, succulent and juicy. The plant emanates a mild, pleasant odour which increases when cut or crushed. It is widely used in making native medicines (Ayurveda, Homeopathy, Unani and Siddha). The aromatic leaves are used for flavouring meat, soups, fish and also is eaten as a vegetable in most tropical countries and Africa. The herb is a traditional folk remedy in India (south), externally it is applied for burns and insect bites; internally it is used as a carminative and to control asthma. It is used as a relief for cold and is said to be very effective in aiding digestion. Daily chewing of a leaf is recommended for all age groups; especially during winter to ward off cold.

Microbes used for this study were collected by vaginal swab method from married, non pregnant women between 18 to 30 years of age complaining of lower abdominal pain related to gynaecology, with mild or profuse white discharge or vaginal irritation from three private hospitals in Madurai , Tamil Nadu , India with the full consent of the patients. Organisms were inoculated in a vial containing sterile Luria broth and were placed in a shaker for $24 \mathrm{~h}$.A sterile cotton bud was dipped in to the Luria broth culture and swabbed on to a sterile Muller-Hinton agar plate aseptically so as to form an even lawn of culture and set aside for 5 min.

Home grown mature leaves of $C$. aromaticus were collected and washed first in sterilized distilled water, followed by washing in mercuric chloride solution $(0.1 \%)$ and again washed in sterilized distilled water , discs measuring $4 \mathrm{~mm}, 7 \mathrm{~mm}, 10 \mathrm{~mm}$ and $13 \mathrm{~mm}$ were cut and washed again in sterilized distilled 
water. Using a sterile forceps a leaf disc was placed on to a neatly microbe swabbed agar plate and gently pressed. The plate was then kept in the same position for $3 \mathrm{~h}$. and then inverted and incubated at room temperature for $24 \mathrm{~h}$. Care was taken that the the placing of leaf disc was done within three $\mathrm{h}$. from the time of collection from the plant. Sterile paper (Whattman filter paper No: 2) discs were used for control, (the only modification that was made was instead of paper discs, leaf discs of different sizes were used) (Bauer et al, 1996).

\section{Results}

Microbes were identified, confirmed and isolated using differential media. The same was subcultured in order to get a pure strain and identity was re-confirmed using bio-chemical tests before proceeding further. Organisms isolated were Candida albicans, Candida krusei, Proteus mirabilis , Escherichia coli, Staphylococcus aureus, Enterococcus faecalis, Klebsiella pneumoniae and Neisseria gonohorreae.

All microbes tested with Coleus aromaticus showed various degrees of activity. Results show that as the size of the leaf disc increases the zone of inhibition of microbe also increases (Table: 1), except for Escherichia coli and Candida krusei. The diameters of inhibition zones were measured in millimeters, the activity values were standardized after a series of tests with different sized discs (Table 1a).

Table1: Antimicrobial activity of Leaf discs of Coleus aromaticus

\begin{tabular}{|c|c|c|c|c|c|}
\hline & \multicolumn{4}{|c|}{ Different diameters of $C$. aromaticus Leaf discs } & $\begin{array}{l}\text { Comparative } \\
\text { over all } \\
\text { average }\end{array}$ \\
\hline Neisseria gonohorreae & 5.67 & 8.93 & 10.11 & 12.32 & 9.25 \\
\hline Enterococcus faecalis & 7.10 & 11.33 & 15.00 & 18.33 & 12.94 \\
\hline Escherichia coli & 7.84 & 16.88 & 16.33 & 21.87 & 15.73 \\
\hline Proteus mirabilis & 9.31 & 14.76 & 17.60 & 21.75 & 15.85 \\
\hline Staphylococcus aureus & 7.33 & 12.66 & 18.33 & 19.29 & 14.40 \\
\hline Klebsiella pneumoniae & 5.80 & 9.35 & 11.89 & 14.81 & 10.46 \\
\hline $\begin{array}{l}\text { Over all antibiotic effect of } \\
\text { leaf } \\
\text { disc in different diameters } \\
{[\mathrm{mm}] \text { for bacteria }}\end{array}$ & 7.17 & 12.31 & 14.87 & 18.06 & - \\
\hline Candida albicans & 9.38 & 15.67 & 21.75 & 21.75 & 17.13 \\
\hline Candida krusei & 10.45 & 15.67 & 23.93 & 20.50 & 17.63 \\
\hline $\begin{array}{l}\text { Over all potential of leaf disc } \\
\text { in different diameters [mm ] } \\
\text { for fungi }\end{array}$ & 9.91 & 15.67 & 22.84 & 21.12 & - \\
\hline $\begin{array}{l}\text { Over all antibiotic effect of } \\
\text { leaf disc in } \\
\text { different diameters [mm ] for } \\
\text { both fungi and bacteria }\end{array}$ & 8.54 & 13.99 & 18.85 & 19.59 & - \\
\hline
\end{tabular}


Table 1a: Activity rating of different sized leaf discs of Coleus aromaticus

\begin{tabular}{llll} 
Disc diameter & No activity & Moderate activity & Highly active \\
\hline $4 \mathrm{~mm}$ & $<7.00$ & $7.00-9.00$ & $>9.00$ \\
$7 \mathrm{~mm}$ & $<10.00$ & $10.00-12.00$ & $>12.00$ \\
$10 \mathrm{~mm}$ & $<13.00$ & $13.00-15.00$ & $>15.00$ \\
$13 \mathrm{~mm}$ & $<16.00$ & $16.00-18.00$ & $>18.00$
\end{tabular}

Figure:1 Comparative effect of different sized leaf discs of Coleus aromaticus on RT microbes
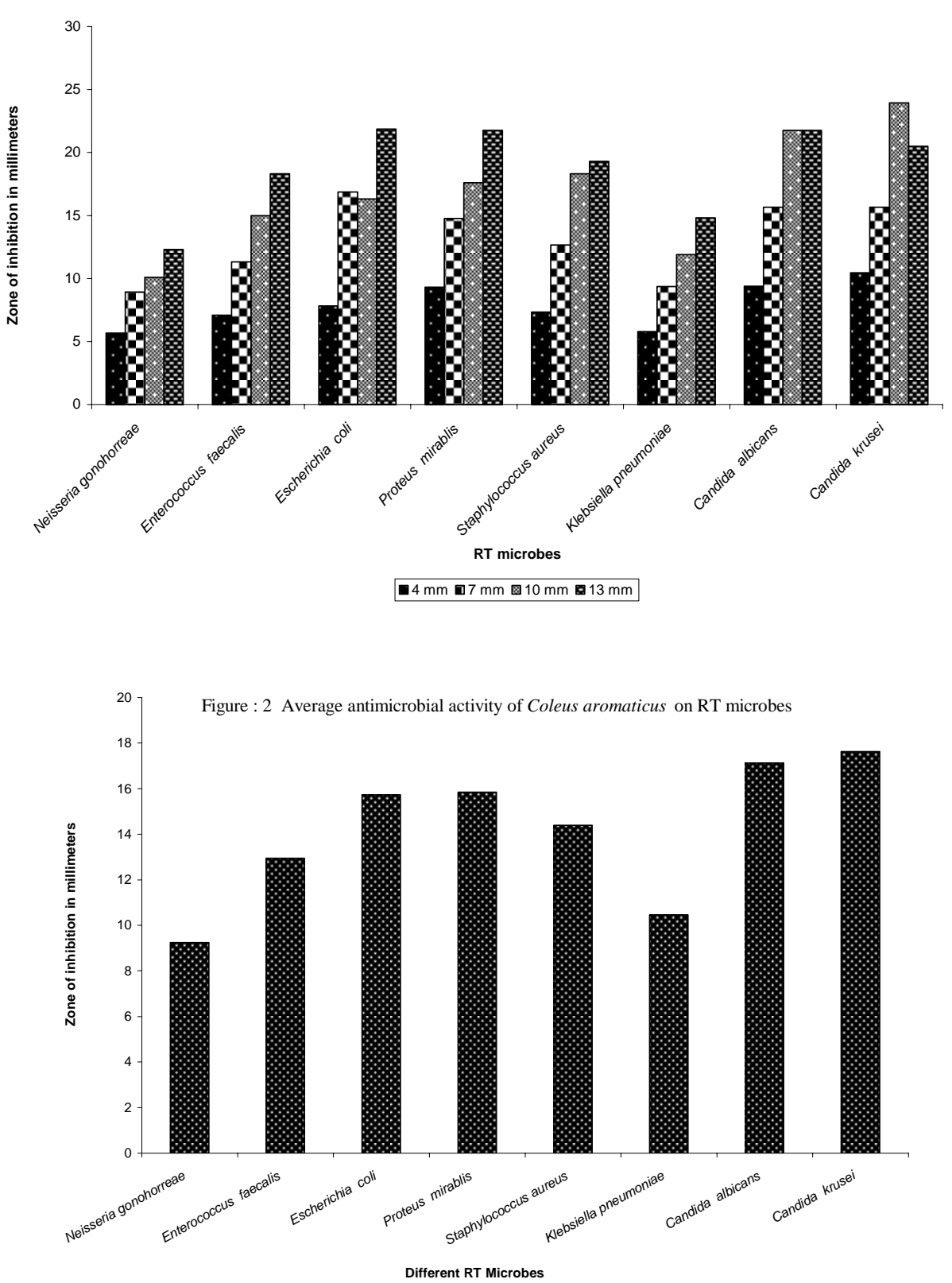
For Candida albicans and Candida krusei, the results were marginally different [Table :1]. For Candida albicans there is an increase in inhibition as the disc size increases but for $10 \mathrm{~mm}$ and $13 \mathrm{~mm}$ the inhibition rate was the same. For Candida krusei there is progressive increase along with the size of the disc, but an apparent dip could be seen for $13 \mathrm{~mm}$, against $10 \mathrm{~mm}$. It was highly active for all other sizes of leaf discs.

On comparing the antibacterial effect of different sized discs of C. aromaticus, for $4 \mathrm{~mm}$ disc Enterococcus faecalis, Staphylococcus aureus and Escherichia coli were moderately active (Figure:1) and for Proteus mirabilis it was highly active. For $7 \mathrm{~mm}$ disc Enterococcus faecalis was moderately active and Escherichia coli, Proteus mirabilis and Staphylococcus aureus were highly susceptible. For $10 \mathrm{~mm}$ disc Enterococcus faecalis alone was moderately active while the rest were highly susceptible. For $13 \mathrm{~mm}$ disc all bacteria were highly susceptible (Figure 1).

As the size of the leaf disc increases the rate of inhibition also increases, except for Escherichia coli $(7 \mathrm{~mm}$ disc $=16.88 \mathrm{~mm}, 10 \mathrm{~mm}$ disc $=16.33 \mathrm{~mm})$ and Candida krusei $(10 \mathrm{~mm}$ disc $=23.93 \mathrm{~mm}, 13$ $\mathrm{mm}$ disc $=20.50 \mathrm{~mm}$ ). Candida krusei had the highest zone of inhibition (Figure 2) followed by Candida albicans, Proteus mirabilis, Escherichia coli, Staphylococcus aureus, Enterococcus faecalis , Klebsiella pneumoniae and the least was found for Neisseria gonohorreae. It is noted that both Neisseria gonohorreae and Klebsiella pneumoniae were not sensitive to this herb. This indicates that C. aromaticus is more effective as an antifungal herb than as an antibiotic agent.

\section{Discussion}

The medicinal properties of plant species have made an outstanding contribution in the origin and evolution of many traditional herbal therapies. These traditional knowledge systems have started to disappear with the passage of time due to scarcity of written documents and relatively low income in these traditions. Over the past few years, medicinal plants have regained a wide recognition due to an escalating faith in herbal medicine in view of its lesser side effects compared to allopathic medicine in addition , the necessity of meeting the requirements of medicine for an increasing human population (Chandra et al, 2006). An ethno botanical survey, on the use of medicinal plants in Kancheepuram district in Tamil Nadu, India revealed that the traditional healers used 85 species of plants distributed in 76 genera belonging to 41 families to treat various diseases. Traditional healers are dwindling in number and there is a grave danger of traditional knowledge disappearing soon since the younger generation is not interested in taking up this traditional healing profession (Chellaiah et al, 2006). Twenty-five to fifty percent of current pharmaceuticals are derived from plants, none are used as antimicrobials. Traditional healers have long used plants to prevent or cure infectious conditions. Western medicine is trying to authenticate their efficacy. Plants are rich in a wide variety of secondary metabolites such as tannins, terpenoids, alkaloids, and flavonoids, which have been found invitro to have antimicrobial properties. Currently, one-quarter to one-half of all pharmaceuticals dispensed in the US are of higher-plant origin; very few are sourced for use as antimicrobials, since people have relied on bacterial and fungal sources for these activities. Since the advent of antibiotics in the 1950`s the use of plant derivatives as antimicrobials has been virtually nonexistent (Marjorie, 1999). Natural-product chemists and microbiologists alike feel that the multitude of potentially useful phytochemical structures which could be synthesized chemically is at risk of being lost irretrievably (Borris, 1996).

Twenty seven varieties of rose flower petals (Anvil sparkes, Ariana 93 , Brass Brand , Baby Talk, Banjaran, Charleston, Class Act, Cabaret, Christian Dior, City of Belfast, Deep Purple, Dearest Peach pink, Harness Marigold, Just Joey, John F Kennedy, Lustige, Maria Callas, Madame Violet, Midas Touch, Orange N Lemons, Paradise, Plain Talk, Red Recke, Summer Holiday, Summer Snow, Vital Sparks and Zorina) were tested for antibacterial activity out of which 19 were found to have moderate to strong antibacterial activity and remaining 8 varieties were found to have weak antibacterial activity. The petals were cut to $10 \mathrm{~mm}$ diameter discs and used. Escherichia coli, Enterococcus faecalis and Klebsiella pneumoniae were weakly sensitive. Pseudomonas aeruginosa, which was resistant to most standard antibiotics, was found to be sensitive to almost all varieties of rose flower petals. Petals of rose flower can be used against infections caused by these microbes. This natural cure can partially replace the use of antibiotics (Tambekar and Mankar, 2006).

If rose flower petals can be used as discs, an attempt was made to use leaves as discs in this work. Leaf used as a disc can be taken to be a preliminary research step in ascertaining the antibacterial or fungal 
activity of a plant, before proceeding further. When a leaf is cut and used as a disc, the leaf cell shrinks to release the contents out which takes time or is done slowly. In this leaf disc method there is sustained (slow) release of the plant juice in to the microbe swabbed medium. This sustained release fairly mimics the action of an antibiotic or fungal disc used in disc diffusion method.

Freeze-dried aqueous extract of C. aromaticus extract clearly established antioxidant potency (Kumaran and Karunakaran , 2006 ). C. aromaticus has been used historically for menorrhagia in Trinidad (Lans , 2006). Hydro-distilled leaves of $C$. aromaticus (collected during May-September from plants growing in India), were analysed by capillary GC and GC-MS. The essential oils contained carvacrol (53-67\%), pcymene (6.5-12.6\%) and gamma-terpinene (5.9-15.5\%) as major constituents. The essential oil produced in September contained higher concentrations of carvacrol and beta -caryophyllene and oxygenated constituents than the essential oil produced in May (Mallavarapu et al ,1999). Native Caribbean plants have been less studied than those from Africa, India and Europe. Achyranthes indica, C. aromaticus, Hibiscus rosa-sinesis, Parthenium hysterophorus and Ruta graveolen, are used for reproductive issues (Cheryl , 2007). C. aromaticus has antioxidant, anticlastogenic and radioprotective potential, which may be of great pharmacological importance (Satish et al, 2006).

Photoactivity was observed from extracts of 10 species belonging to 10 families, including Convolvulaceae, Lamiaceae and Rhamnaceae, where photoactivity has not been previously reported. This is the first study where plants from highly stressful serpentine environments have been tested for antimicrobial activity. Plants from serpentine environments may have altered antimicrobial activities compared to their relatives from non-serpentine environments (Nishanta et al , 2002). The in vitro antimicrobial activity of the essential oils of $C$. aromaticus and $C$. zeylanicus was tested against seven bacteria (Bacillus megaterium, B. subtilis, Escherichia coli, Staphylococcus aureus, Proteus vulgaris, Pseudomonas aeruginosa, and Xanthomonas campestris) and eight fungi (Aspergillus niger, A. parasiticus, Rhizopus oryzae, Rhizoctonia oryzae-sativae, Colletotrichum musae, Fusarium solani, Candida albicans, and Alternaria brassicicola). The oil of $C$. zeylanicus had slightly higher inhibitory activity against a wide spectrum of bacteria and fungi (Deena et al., 2002 ).

It is obvious from the results that $C$. aromaticus is a fairly good antibiotic and a very effective antifungal herb. Its inhibitory action against, Candida krusei was the highest followed by Candida albicans with a marginal difference (Table 1). The following is the order of susceptibility for Coleus aromaticus , Proteus mirabilis, Escherichia coli, Staphylococcus aureus, Enterococcus faecalis . Klebsiella pneumoniae and Neisseria gonohorreae were found to be least susceptible. Further research C. aromaticus will definitely show its potential as an antimicrobial agent.

\section{Conclusion}

From this study it is clear that $C$. aromaticus indeed exhibits a potent anti-fungal and moderate anti-bacterial activity. More research needs to be done to unravel the inhibitory effect of this plant. Since this herb had been used for ages traditionally and effectively, it is presumed that side effects should be less. Use of herbs by Indian (south) community is a well known fact; there is a treasure of herbs that we use daily in our food or in other forms customarily, even without knowing their medicinal benefits. Such use of plant material has always been a tradition, mostly community based that is passed on from one generation to another. In general, lesser known or used herbs and plant materials have to be researched further to study their medicinal properties especially their antibiotic nature. This will enable the use of our own local, rich plant heritage as effective medicines with probably fewer side effects.

\section{References}

1. Bauer A W ., Kirby W. , Sherris M and Tureck M . (1996). Antibiotic susceptibility testing by a standardized single disc method. Am J Clin Pathol .,Vol 45 ., 493-496.

2. Borris R P. (1996). Natural products research: perspectives from a major pharmaceutical company. J Ethnopharmacol.51:29-38

3. Chandra Prakash Kala, Pitamber Prasad Dhyani and Bikram Singh Sajwan. (2006) .Developing the medicinal plants sector in northern India: challenges and opportunities. Journal of Ethnobiology and Ethnomedicine . 2:32. 
4. Chellaiah Muthu ., Muniappan Ayyanar ., Nagappan Raja and Savarimuthu Ignacimuthu. (2006). Medicinal plants used by traditional healers in Kancheepuram District of Tamil Nadu. Indian Journal of Ethnobiology and Ethnomedicine 2:43.

5. Cheryl Lans . ( 2007) . Ethnomedicines used in Trinidad and Tobago for reproductive problems. J Ethnobiol Ethnomedicine. 3: 13.

6. Deena M. J., Sreeranjini K and Thoppil J E . (2002 ). Antimicrobial screening of essential oils of Coleus aromaticus and Coleus zeylanicus .International Journal of Aromatherapy 12(2): 105-107 .

7. Farnsworth N.R and Morris R W. (1976) .Higher plants: the sleeping giant of drug development. Am. J. Pharm. 46.

8. Farnsworth N R ., Akerele O. , Bingel A S., Soejarta D D and Eno Z . (1985). Medicinal plants in therapy. Bull World Health Organ. 63 (6) :965-981.

9. Friedrich EG Jr. (1985) . Vaginitis. Am J Obstet Gynecol. 152 (3):247-51.

10. Ignacimuthu S ., Ayyanar M and Sankara Sivaraman (2006).Ethnobotanical investigations among tribes in Madurai District of Tamil Nadu (India).Journal of Ethnobiology and Ethnomedicine .2:25.

11. Indu M N ., Hatha A A M ., Abirosh C ., Harsha U and Vivekanandan G .( 2006). Antimicrobial activity of some of the south Indian spices against serotypes of Escherichia coli, Salmonella , Listeria monocytogenes and Aeromonas hydrophila. Brazilian Journal of Microbiology. 15;7-8382.,37:153-158.

12. Jasmin Helen Prasad ., Sulochana Abraham ., Kathleen M. Kurz ., Valentina George., Lalitha M K ., Renu John ., Jayapaul M N R ., Nandini Shetty and Abraham Joseph, (2005): Reproductive Tract Infections among Young Married Women in Tamil Nadu, India International Family Planning Perspectives. 31 (2):73-82

13. Kumaran A and Karunakaran R. J. (2006). Antioxidant and free radical scavenging activity of an aqueous extract of Coleus aromaticus .Food Chemistry 97 (1): 109-114

14. Lans C. (2006).Creole remedies of Trinidad and Tobago. Lulucom. http://www.lulu.com/content/302210 .

15. Mallavarapu G R ., Laxmi Rao and Srinivasaiyer Ramesh .(1999 ).Essential oil of Coleus aromaticus [Benth] from India . Journal of Essential Oil Research 11(6):742-744.

16. Marjorie Murphy Cowan . (1999 ). Plant Products as Antimicrobial Agents. Clin Microbiol Rev.12 (4): $564-582$.

17. Nishanta Rajakaruna ., Harris C S and Towers G H N ( 2002 ). Antimicrobial activity of plants collected from serpentine outcrops in Sri Lanka . Pharmaceutical Biology . 40 (3): 235-244 .

18. Satish Rao B S ., Shanbhoge R ., Upadhya D., Jagetia G C ., Adiga S K ., Kumar P., Guruprasad K and Gayathri P .(2006). Antioxidant, anticlastogenic and radioprotective effect of Coleus aromaticus on Chinese hamster fibroblast cells (V79) exposed to gamma radiation . Mutagenesis .21(4):237-242.

19. Spiegel C A .(1991). Bacterial Vaginosis. Clin Microbiol Rev. 4 (4): 485-502.

20. Srinivasan D ., Sangeetha Nathan ., Suresh T and Lakshmanaperumalsamy P. (2001).Antimicrobial activity of certain Indian medicinal plants used in folkloric medicine . J.Ethnopharmacol.,74. 217 -220/

21. Tambekar D H and Mankar S S . (2006 ). Studies on antimicrobial spectrum of rose petals against bacterial pathogens .Asian J of Microbiol Biotec Env sci . Vol 8, No (2):367-372.

22. Veeramuthu Duraipandiyan., Muniappan Ayyanar and Savarimuthu Ignacimuthu. (2006). Antimicrobial activity of some ethnomedicinal plants used by Paliyar tribe from Tamil Nadu, India. BMC Complementary and Alternative Medicine .6:35. 